\title{
Moisture Content Effect on Ultrasonic Velocity in Goupia Glabra
}

\author{
Fabiana Goia Rosa de Oliveira ${ }^{\mathrm{a} *}$, Marcela Candian ${ }^{\mathrm{b}}$, Francieli F. Lucchette ${ }^{\mathrm{b}}$, \\ José Luis Salgon ${ }^{\mathrm{b}}$, Almir Sales ${ }^{\mathrm{b} *}$ \\ ${ }^{a}$ University of São Paulo, São Carlos - SP, Brazil \\ ${ }^{\mathrm{b}}$ Department of Civil Engineering, UFSCar, Federal University of São Carlos, São Carlos - SP, Brazil
}

Received: June 10, 2003; Revised: January 10, 2005

\begin{abstract}
This paper discusses the application of ultrasound waves on a Brazilian hardwood, Goupia glabra, to evaluate the sensitivity of the ultrasonic technique to the moisture content in wood. The velocity of ultrasonic wave is sensitive to the material's quality-determining factors; hence, this technique is an important industrial tool to improve the quality control of processes. The nature of the response of velocity of sound to changes in moisture content led us to conclude that moisture gradients during drying exert a dominating effect. The ultrasonic velocity was measured both parallel and perpendicular to the fibers of Goupia glabra during drying from green to 6\% moisture content. The results of this study showed that velocity of ultrasonic waves is sensitive to changes in moisture content of lumber during drying. The velocity under dry conditions was always higher than the velocity under more humid conditions, in both directions of propagation.
\end{abstract}

Keywords: ultrasonic velocity, moisture content, wood

\section{Introduction}

In order to relate the anatomical structure to the acoustic behavior of wood it is necessary to understand some mechanisms which, during the propagation of vibration, would separate the cells and allow them to act independently. The wood structure can be considered as a rectangular system of cross-homogeneous closed tubes embedded in a matrix. The longitudinal orientation of tracheids or fibers is partially disturbed by medullary rays. The ultrasonic energy injected into a fibrous material couples several modes on each fiber. The physical properties of the cellular wall, density, rigidity modulus and the shape and size of the fibers affect the transmitted ultrasonic field. Each structural element acts independently as an elementary resonator. The spatial distribution of velocities and frequencies that matched the frequency of the natural fibers could explain the acoustical behavior of wood as illustrated by its overall parameters ${ }^{1}$.

The interfacial discontinuities, cracks, porosity or density variations may be detected by nondestructive techniques such as ultrasound. This method can help in the understanding of material behavior under different environmental conditions, but difficulties still remain for detection and for quantitative description of structural discontinuities and defects. It is important to relate the nondestructive measurements to the mechanical properties of wood and wood-based composities. The basis of such relations is the dependence of the interfacial strength, on one or more mechanical characteristics related to the nature and morphology of defects ${ }^{2}$.

In regard to the fiber length, it can be stated that the longer the fibers the greater the velocity of wave propagation. This due the greater continuity of crystalline and elastic material in the longitudinal direction and explains the occurrence of higher wave velocities in conifers than in dicotyledons for samples with constant moisture content, even with the predominance of apparently mower densities in conifers ${ }^{3}$.

Ultrasonic testing consists effectively of the propagation of low amplitude waves through a material to measure either or both the time of travel and any change of intensity for a given distance. Applications include distance gauging, flaw detection and measuring parameters which are related to the material structure.
The propagation of ultrasonic waves in wood depends mainly on the mechanical properties of the cell wall. The cell wall density is reasonably constant, but the modulus of elasticity varies owing to variations in the cell wall structure; hence, one can expect a range of values for the velocity propagation property ${ }^{1}$.

The value of the velocity of sound in a material depends on the appropriate elastic modulus, which is characteristic of the nature of the vibrational stress applied to it and also on the density of the material. It is assumed that the amplitudes of the stresses are sufficiently low to allow for purely elastic conditions ${ }^{4}$.

The appropriate elastic modulus governing the value of the velocity of sound depends on the type of waves which are propagated and the relative values of the wavelength and cross-sectional dimensions of the irradiated object. For compression waves propagated along the axial direction in a bar having lateral dimensions small compared with the wavelength, the appropriate elastic modulus is the Young modulus, and the velocity is given by:

$$
\mathrm{V}=(\mathrm{E} / \rho)^{1 / 2}
$$

Where $\rho$ is the density (for solid rods). Because there are no lateral constraints imposed by the material of the rod, compressions and tensions in the axial direction give rise to expansions and contractions, respectively, in the plane of the cross-section by amounts determined by Poisson ration, $v$, as defined by:

$$
v=-\varepsilon_{2} / \varepsilon_{1}
$$

Where $\varepsilon_{1}$ and $\varepsilon_{2}$ are respectively the axial and linear cross-sectional strains.

The principal wave types for measuring wood properties are bulk waves (longitudinal or transverse) and surface waves (Rayleigh, Lamb and Love waves). The waves are characterized by the direction of propagation and by the particle motion. For longitudinal waves the particle trajectory is in the direction of propagation; for transverse waves the particle motion is perpendicular to the direction of propagation ${ }^{1}$. 
In Rayleigh surface waves, the vibrations are confined to the neighborhood of the surface and the acoustic energy penetrates the solid by a distance of not greater than one or two wavelengths; for this reason the attenuation of surface waves is considerably less than that for bulk waves. Another type of surface waves is Love waves for which the polarization lies in the plane of the surface but perpendicular to the direction of propagation. However, they are an upper frequency limit of a few thousand hertz and are normally encountered only with seismic applications ${ }^{4}$.

In dispersive media the ultrasonic velocity is dependent on frequency, and boty phase and group velocities can be measured. The relationships between group velocity $(v)$ and phase velocity $(V)$ can be summarized in the relation:

$$
\mathrm{V}=\mathrm{V} /(1-f / \mathrm{V} d \mathrm{~V} / d f)
$$

The phase velocity is:

$$
\mathrm{V}=\omega / k=f^{*} \lambda
$$

Where $\omega=2 \pi f$ and $f$ is the frequency, the propagation constant is $\mathrm{k}=2 \pi / \lambda$, and $\lambda$ is the wavelength.

The factors that influence the propagation of ultrasonic waves in wood are physical properties of the substrate, geometrical characteristics of the species (macro and microstructures), conditions of the medium (temperature, humidity) and the procedure utilized to take the measurements ${ }^{5}$ (frequency and sensitivity of the transducers, their size, the position and dynamic characteristics of the equipment).

Physical properties of wood such as electric condutivity, mechanical strength and elasticity are strongly dependent on the amount of water included ${ }^{6}$. Wood hold water in two different ways. When they are very dry, they absorb water within the wall of wood cells. Water molecules are bonded to hemicellulose or cellulose of the amorphous region of wood substance. The microscopic absorption mechanism continues up to the FSP (fiber saturation point), at which point all the possible sites are occupied and the cell is saturated with the absosbed water. Above the FSP, microscopic vacant spaces in the cells work as containers to retain free water.

The ultrasonic velocity through wood varies with moisture content even above the fiber saturation point ${ }^{7}$. The nature of the response of ultrasonic velocity to moisture content changes led us to conclude that moisture gradients during drying exert a dominating effect.

Researchers ${ }^{1,6-8}$ conducted several studies on the relationship between ultrasonic velocity and moisture content. Most of these studies were conducted in an arrangement in which the dry shell and wet core were ordered in serial form along the direction of sound transmission. As applied to kiln control, this arrangement would correspond to measuring ultrasonic velocity through the thickness of a wide board during drying. A range of relationships was found depending on species. In most species, the increase in velocity from green to the fiber saturation point was not very significant. However, the increase in velocity from fiber saturation to $0 \%$ moisture content was much greater?

With a low content $(U<18 \%)$, when the water is present in the cell walls as bound water, the ultrasonic pulse is scattered by the wood and by cell boundaries. The side units of $\mathrm{OH}$ or other radicals of the cellulosic material may reorient their position under the ultrasonic stress. In this case the attenuation mechanism related to the cellulosic cell wall material is probably the most important. With higher moisture content but under the fiber saturation point, the scattering at cell boundaries could be the most important loss mechanism. When the fiber saturation point is exceeded and free water is present in cellular cavities, the material's porosity takes over as a predominant factor in ultrasonic scattering ${ }^{1}$.
The purpose of this study is to investigate the ultrasonic technique's sensitivity to the moisture content of wood, aiming to establish the relationship between the two characteristics and to analyze the feasibility of applying this method to estimate moisture content during drying of Goupia glabra.

\section{Experimental Procedure}

Six boards of Goupia glabra having length of $750 \mathrm{~mm}$ and crosssection of $25 \times 300 \mathrm{~mm}$ were dried from green $(60 \%)$ to $6 \%$ moisture content. The experimental procedure consisted of two measurements: moisture content and ultrasonic velocity. Although the literature suggests that the parallel arrangement will result in higher sensitivity in velocity of sound with moisture content above the fiber saturation point, in this study, both situations were analyzed.

The ultrasonic technique used is based on the transmitting pulse method. The propagation velocity was determined by measuring the specimen length and the travel time (time-of-flight) of the ultrasonic wave. The longitudinal ultrasonic pulse was generated by a Sylvatest (Concept Bois) ultrasonic apparatus, operating at a frequency of $22 \mathrm{kHz}$ (Figure 1). Ultrasonic measurements were taken both, perpendicular and parallel to the fibers, using longitudinal ultrasonic waves (Figure 2).

Medicinal gel was applied at the tops of the tested boards to ensure good contact between the wood and the transducers. This gel provides better transmission of the ultrasonic wave at the interface, preventing interferences in the signal. Many bonding media have been used over a wide range of temperatures and moisture content. Methods of creating the bond vary with the testing conditions. With hardwoods, when the specimen is large enough (structural dimensions), the transducer may be screwed into the sample and no coupling medium is needed.

The boards were soaked in water for a time, after that, they were dried in air to reduce the moisture until $12 \%$. Finally, the boards were dried until $6 \%$ in a kiln. For every variation of moisture content about $2 \%$, the longitudinal ultrasonic measurements were taken, from green to $6 \%$ moisture content.

\section{Results and Discussion}

The average green and dry velocities are given in Table 1 .

Both velocities, parallel and perpendicular to the fibers were strongly and continuously affected by the variation of moisture

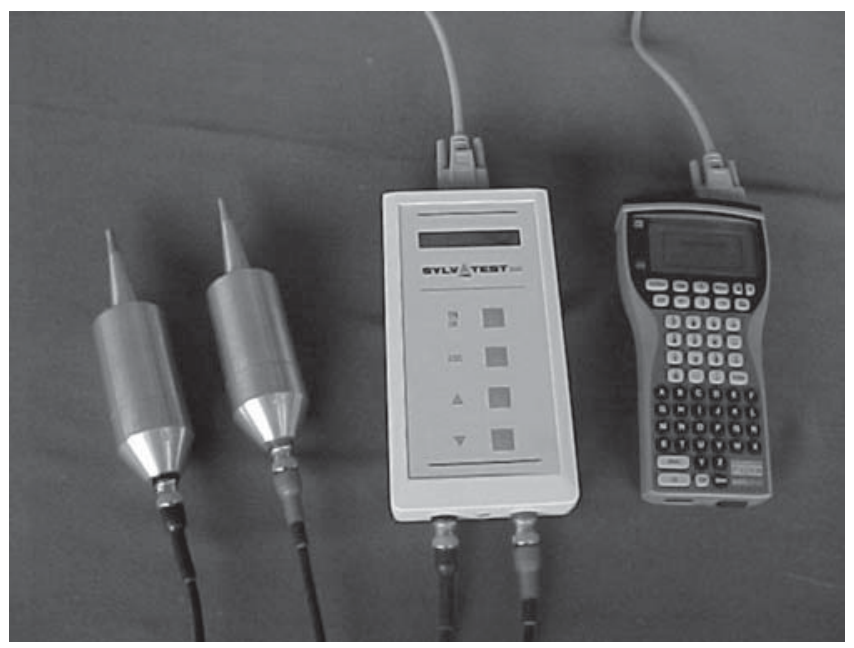

Figure 1. Sylvatest ultrasonic device. 
content. The maximum velocity was measured for the longitudinal direction when the specimens were dried (6\% moisture content). The minimum velocity was measured for the transverse direction when specimens were under more humid conditions (60\% moisture content).

The values of velocity corresponding to the measurements obtained from the transverse section of the specimen are less affected by the modification of the moisture content. The variation of velocities values of the velocity parallel to the fibers during drying showed an increase of $11.4 \%$ and in the perpendicular direction produced a $7.6 \%$ increase in longitudinal velocity.

The longitudinal ultrasonic velocity parallel to the fibers decreased with increasing moisture content. An inflection point of the curve existed around the fiber saturation point. The results showed that the effects of moisture content under FSP on velocity were stronger than that over the FSP. It seems that for the longitudinal direction, the moisture content over the FSP does not influence the ultrasonic velocity.

The ultrasonic velocity perpendicular to the fibers also decreased

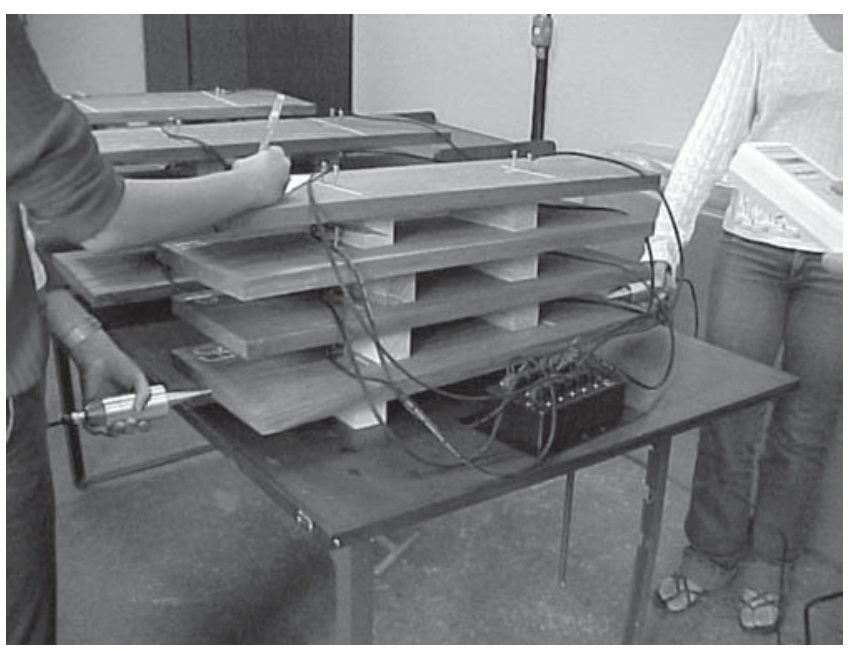

(a)

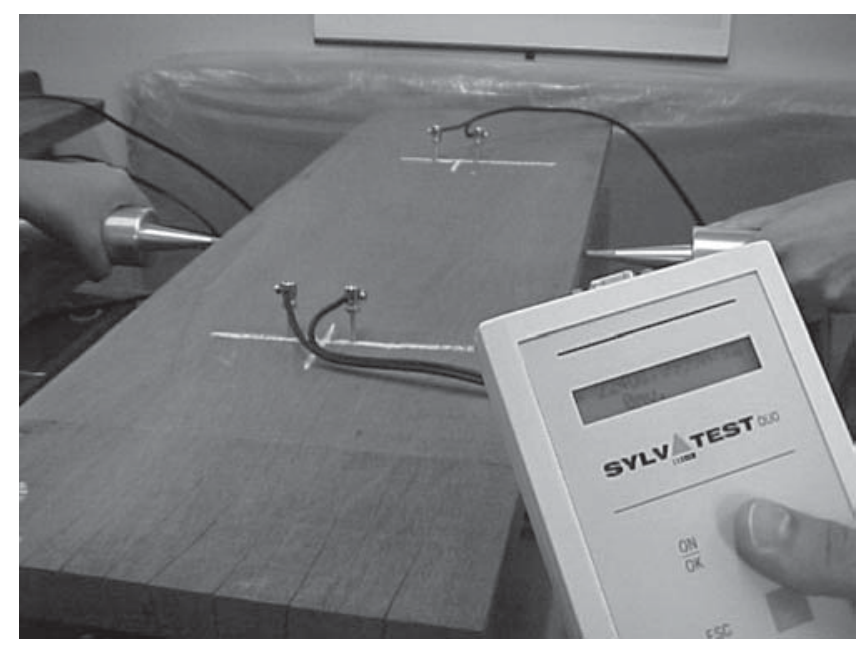

(b)

Figure 2. Ultrasonic measurements: a) parallel to the fibers; b) perpendicular to the fibers. with increasing moisture content; however, as suggested in the literature, the nature of this response was not as strong as it was in the longitudinal velocity. During drying, the ultrasonic velocity was faster in the shell than in the wet core, which influenced the overall velocity measured through the board.

The relationships between ultrasonic velocity parallel and perpendicular to the fibers and moisture content of the boards are shown in Figures 3 and 4.

The velocities were transformed into relative velocities. The relationship between relative velocity and moisture content, both perpendicular and parallel to the fibers, was characterized by linear regression (Figures 5 and 6).

The coefficient of determination obtained in the longitudinal direction $\left(r^{2}=0.97\right)$ was more significant than the coefficient in the transversal direction $\left(r^{2}=0.78\right)$, indicating a better adjustment between the variation of the velocity and the variation of the moisture content in the longitudinal direction.

The longitudinal orientation of cells along the longitudinal axis

Table 1. Average ultrasonic velocity for green and dry Goupia glabra wood.

\begin{tabular}{lrrrr}
\hline & \multicolumn{2}{c}{$\begin{array}{c}\text { Parallel to fibers } \\
(\mathrm{m} / \mathrm{s})\end{array}$} & \multicolumn{2}{c}{$\begin{array}{c}\text { Perpendicular to } \\
\text { fibers }(\mathrm{m} / \mathrm{s})\end{array}$} \\
& Green & \multicolumn{1}{c}{ Dry } & Green & \multicolumn{1}{c}{ Dry } \\
\hline Average & 4162.0 & 4637.0 & 1099.0 & 1183.0 \\
Minimum & 3946.0 & 4533.0 & 1077.0 & 1140.0 \\
Maximum & 4291.0 & 4778.0 & 1111.0 & 1260.0 \\
Standard deviation & 130.6 & 85.9 & 12.4 & 45.4 \\
$\begin{array}{l}\text { Coefficient of varia- } \\
\text { tion (\%) }\end{array}$ & 3.1 & 1.8 & 1.1 & 3.8 \\
\hline
\end{tabular}

Parallel to the fibers

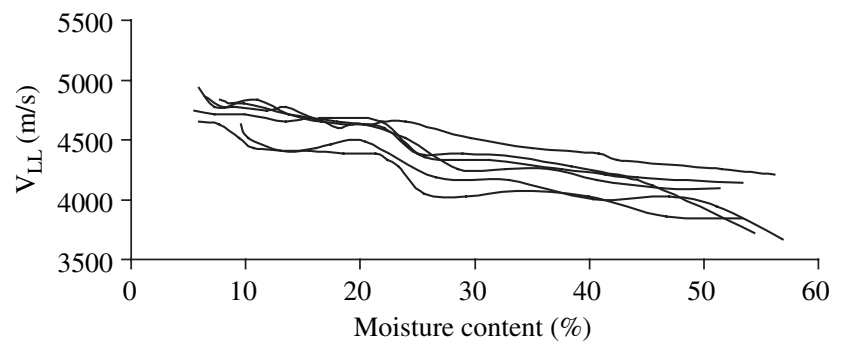

Figure 3. Relationship between the moisture content and the longitudinal velocity measured parallel to the fibers.

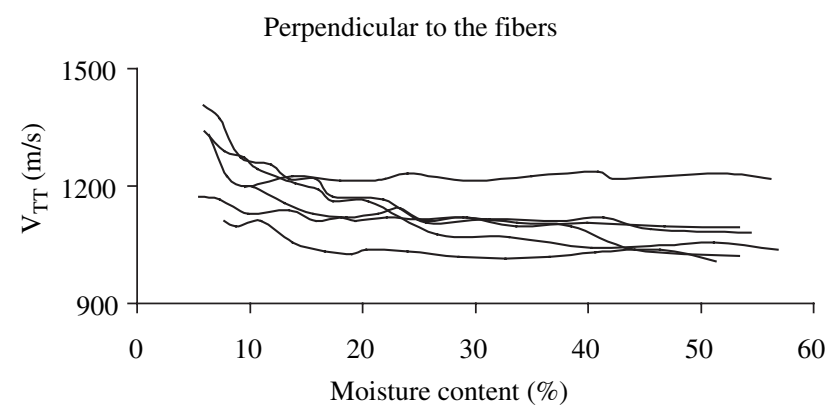

Figure 4. Relationship between the moisture content and the velocity measured perpendicular to the fibers. 


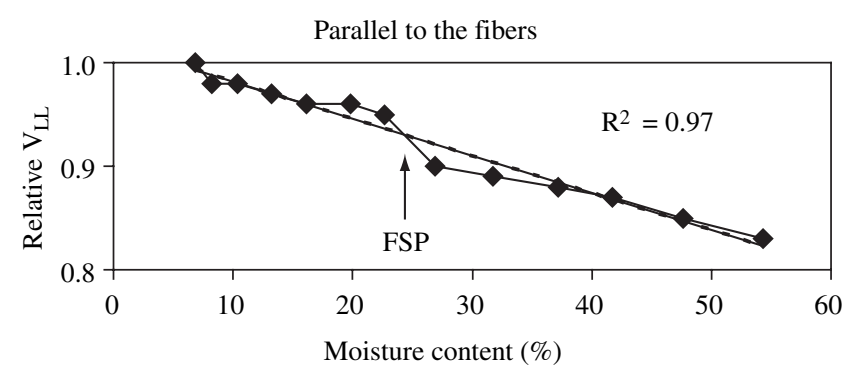

Figure 5. Relative longitudinal velocity parallel to the fibers $v s$. the moisture content.

is the best explanation of the specific ordering of velocity values as cells walls provide a continuous wave path. In the longitudinal direction, the dissipation of acoustic energy probably takes place at the limit of fibers.

\section{Conclusions}

The results of this study demonstrated that ultrasonic velocity is sensitive to changes in moisture content of Goupia glabra. The longitudinal velocity under dry conditions was always higher than the velocity under more humid conditions, in both directions: parallel and perpendicular to the fibers.

The parallel and perpendicular ultrasonic velocities decreased as the moisture content increased. During drying, the velocity perpendicular to the fibers displayed a slighter increase with equilibrium moisture content than it did parallel to the fibers, indicating that the sensitivity of ultrasonic measurement was greater in the longitudinal velocity.

The coefficient of determination obtained in the longitudinal direction $\left(r^{2}=0.97\right)$ was very significant, allowing us to state that the variation in velocity and moisture content in the longitudinal direction is better adjusted.

The results of this study allow us to state that the nondestructive method using ultrasound can be used to reliably evaluate the mechanical properties of tropical wood with structural dimensions. Hence, this nondestructive method can be employed in the classification of structural elements of wood.

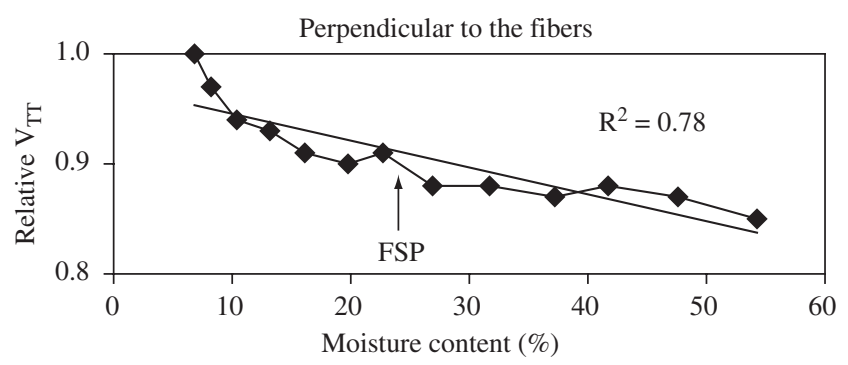

Figure 6. Relative velocity perpendicular to the fibers $v$ s. the moisture content.

\section{Acknowledgements}

The authors thank FAPESP and CNPq for its financial support, and also LaMEM and LMC for their help in developing this study.

\section{References}

1. Bucur V. Acoustics of wood. New York: CRC Press Inc; 1995.

2. Bucur V. Nondestructive characterization and imaging of wood. New York: Springer; 2003.

3. Oliveira FGR, Campos JAO, Sales A. Ultrasonic measurements in brazilian hardwood. Materials Research. 2002; 5(1):51-55.

4. Blitz J, Simpson G. Ultrasonic methods of non-destructive testing. New York: Chapman \& Hall; 1996.

5. Kabir MF, Schmoldt DL, Schafer ME. Time domain ultrasonic signal characterization for defects in thin unsurfaced hardwood lumber. Wood and Fiber Science. 2002; 34(1):165-182.

6. Simpson WT, Wang X. Relationship between longitudinal stress wave transit time and moisture content of lumber during kiln-drying. Forest Products Journal. 2001; 51(10):51-54.

7. Wang X, Chuang S. Experimental data correction of the dynamic elastic moduli, velocity and density of solid wood as a function of moisture content above the fiber saturation point. Holzforschung. 2000; 54(1):309314.

8. Sandoz JL. Moisture content and temperature effect on ultrasound timber grading. Wood Science and Technology. 1993; 27(1):373-380. 